\title{
Safe Spine Surgery
}

S Venkatesh Babu

\author{
Abstract \\ Presently, the surgical treatment is prescribed for most of the spine diseases and fractures. The complications after spine surgery are also \\ increasing. This review piece of writing updates the applied anatomical knowledge and operative skills necessary for the surgeon who intends \\ to surgically treat the spine in a safe manner. \\ Keywords: Injury, Safe, Spine, Surgery, Trauma. \\ Journal of Postgraduate Medicine, Education and Research (2021): 10.5005/jp-journals-10028-1407
}

\section{INTRODUCTION}

The field of spine surgery has evolved appreciably over the past three decades with many advances. Spine surgery includes many common surgical procedures to treat the pathologies causing radiculopathy, myelopathy, instability caused by degeneration or trauma, infection, and tumors. Surgical strategies include decompression of neural elements and stabilization when necessary either through an anterior, posterior, or combined approach. There is a reported $25 \%$ complication after a cervical procedure, $17 \%$ in thoracic, and $32 \%$ in lumbar spine surgical procedures. Deep venous thrombosis and pulmonary embolism are also potential complications of spinal surgery. Surgeons need to be aware of all possible problems with each step of the surgery and the methods to avoid and manage them efficiently. ${ }^{1-4}$

Minimally invasive spine surgeries and vertebral augmentation procedures for the osteoporotic vertebral compressive fractures are also been widely done today. Major complications have been noted after these surgical treatment including the cement leaks in vertebroplasty and other grievous complications ranging from 48 to $74 \%$ after spine surgeries. Sadly, wrong-site surgery in spine operations had also been reported all over the world.,

Spinal trauma often results in a complex interaction of injuries to the musculoskeletal and nervous systems. The combination of unique biomechanical and neurological considerations provides an exceptional challenge to the surgeon managing the spinally injured patient. Advanced surgeries are being done for the spinal trauma. The complications after spine fracture surgery are considerably increasing by instrumentations and had been extensively reported. ${ }^{2,6,7}$

Emergency decompression in acute cervical spinal cord injury remains reasonable practice option today and has to be performed safely. However, optimal timing of surgery is a result of a complex range of variables related to the institution, the patient, and the surgical team. In polytrauma situations, life-threatening injuries need to be treated immediately followed by the salvage of threatened limb. If decompression of the spinal cord is advocated, earliest possible intervention may be desirable. ${ }^{8,9}$

This review article brings up-to-date information, the applied anatomical knowledge, and operative expertise necessary for the spine surgeons who desire to operate and instrument the spine in a safe way.
Department of Orthopedics and Trauma Surgery, Sri Sakthi Hospital, Tirunelveli, Tamil Nadu, India

Corresponding Author: SVenkatesh Babu, Department of Orthopedics and Trauma Surgery, Sri Sakthi Hospital, Tirunelveli, Tamil Nadu, India, Phone: +91 9843057118, e-mail: drsvbabu@hotmail.com

How to cite this article: Babu SV. Safe Spine Surgery. J Postgrad Med Edu Res 2021;55(4):171-176.

Source of support: Nil

Conflict of interest: None

\section{Principles of Safe Spine SURGERY $1-4,6,7,10-17$}

The surgeon must possess the detailed anatomical understanding in spine surgical approaches and precise skills for the safe surgery (Fig. 1). Marking the surgical site with precise identification of the level of vertebra and the accurate instrumentation technique are all needed for patient safety in spine surgery.

The anesthesia and proper patient positioning in addition play a vital role in the safety of the patient. The supportive anesthetist colleague who is able to produce a controlled hypotension in reducing the blood loss and effectively managing other physiological factors will yield good results. There are several general complications that happen in spinal surgery following anesthesia and positioning, bone grafting, wound infection and discitis, cervical traction, dural tear, and cerebrospinal fluid (CSF) leak. These factors must be acknowledged with great precaution. There are indeed specific complications after certain spinal procedures as discussed comprehensively below.

\section{Preventing General Complications in SPINe Surgery}

- The reported incidence of postoperative respiratory compromise varies from 0 to $14 \%$. Avoiding prolonged prone position, manual inline axial stabilization with fiber optic nasal or oral endoscopy can reduce risk of spinal cord injury during tracheal intubation. Patients with cervical instabilities must be given paramount care in providing general anesthesia.

(-) The Author(s). 2021 Open Access This article is distributed under the terms of the Creative Commons Attribution 4.0International License (https://creativecommons. org/licenses/by-nc/4.0/), which permits unrestricted use, distribution, and non-commercial reproduction in any medium, provided you give appropriate credit to the original author(s) and the source, provide a link to the Creative Commons license, and indicate if changes were made. The Creative Commons Public Domain Dedication waiver (http://creativecommons.org/publicdomain/zero/1.0/) applies to the data made available in this article, unless otherwise stated. 


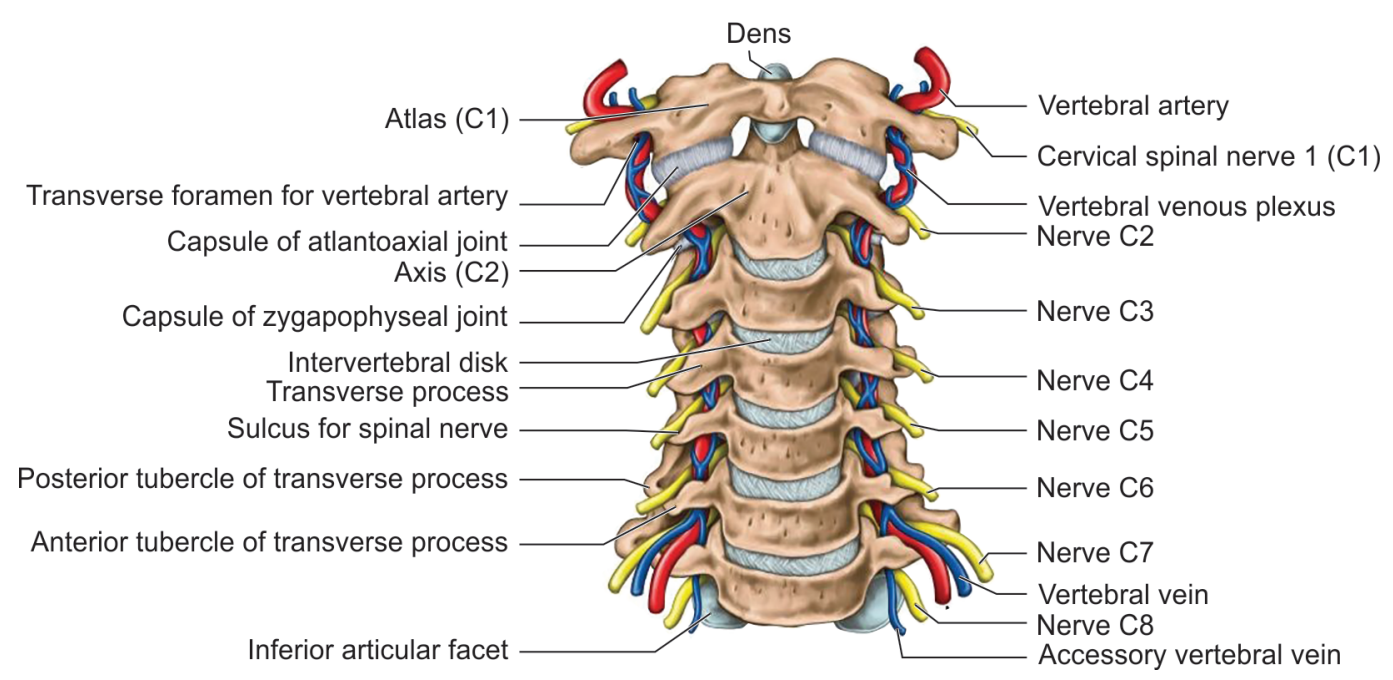

Fig. 1: Cervical spine with close neurovascular structures

- Maintaining adequate blood pressure is important for neural perfusion. At the same time, controlled hypotension reduces blood loss and facilitates spine surgical exposure.

- Positioning the patient is important to permit access to the surgical field and is necessary to avoid pressure areas to iatrogenic injuries. Areas of concern include the face, iliac crests, lower ribs, patella, and the anterior region of ankle joints.

- Excessive traction with the tape or extension of shoulders below the coracoids process should be avoided to prevent compression of the brachial plexus. Hanging the arm over the edge of the operating table can injure the radial nerve. This should also be avoided. Injury to the common peroneal nerve can occur if the legs are placed in abduction and external rotation, which can cause pressure over the fibular head. Adequate padding under pressure points can prevent skin and peripheral nerve complications especially in prolonged prone positioning.

- Adequate head and neck control is mandatory to prevent damage to the spinal cord, such as skeletal traction via Halo ring or Mayfield tongs. Being in a prone position may increase intraocular pressure, which will lead to ischemia, decreased perfusion pressure, and blood supply to the retina. This can result in ischemic optic neuropathy, blindness, and occipital stroke. Pressure over the eyeballs due to Mayfield headrest or Gardner Wells tongs should be avoided. Arms are kept by the side and shoulders are usually pulled distally and taped to the operating table to facilitate lateral radiograph.

- Local complications of cervical traction pins include tract infection, dura penetration leading to leakage of CSF, brain abscess and meningitis, propagation of fractures in presence of skull fractures, loss of fixation, complications of hanging weights, arterial injuries, overdistraction and related disk protrusion, missed distraction type cervical injuries, and pin malpositioning. Injury to the temporal artery can also occur from the traction pins due to direct laceration or pin site invasion. The ideal pin site is at thickest skull bone located above external auditory meatus bilaterally. The area directly anterior and superior to the ear tragus should be avoided.

- Overdistraction is dangerous in occipitocervical dislocations, odontoid fractures and hyperextension, and distraction injuries. Cranial nerve injury involving the abducens, glossopharyngeal, vagus, and hypoglossal nerves has also been reported to occur after traction with an incidence of $0.07 \%$.

- The risk of durotomy during laminectomy is 0.3 to $13 \%$ and can be up to $18 \%$ with revision surgery. Risk factors include old age, thin dura as a result of chronic compression, ossification of the ligamentum flavum, synovial cysts, and scarring from previous surgeries. The ligamentum flavum and posterior longitudinal ligaments are helpful barriers during decompression and these soft tissues should be carefully elevated after the overlying bone is removed.

\section{Anterior Cervical Spine Surgery \\ Preventing Recurrent Laryngeal, Superior Laryngeal, and Hypoglossal Nerve Injuries}

- Safe dissection is advised in any sided approach (right/left). Avoiding the lateral ligature of inferior thyroid vessels will safeguard the recurrent laryngeal nerve injury.

- The sharp-toothed retractors are advised and placing under the longus colli muscle belly and avoidance of trachea-esophageal groove will prevent vocal cord paralysis.

- The endotracheal tube accounts for $11.2 \%$ of vocal cord paralysis. Too much inflation of the tube must be avoided since it will produce retraction pressure against the larynx in causing nerve injury.

- Reaching upper spine (C2-C4) needs much care in preventing superior laryngeal nerve and hypoglossal nerve injury since these nerves may resemble a blood vessel.

\section{Preventing Esophageal Injury}

- As discussed above, the retractors must be carefully placed as mentioned and displacement anteromedially can compress the trachea and esophagus medially. This can be avoided by intermittent release of the retractors.

- However esophageal perforation has an incidence of 0.2 to $1.15 \%$. To avoid this, blunt finger dissection is recommended for exposures below the superficial cervical fascia. Also proud screw fixation must be avoided since the posterior esophageal 
mucosa is very delegate. Perforation of esophagus is a nasty complication; hence, it may lead to various dangerous complications like mediastinitis, retropharyngeal abscess, pneumonia, and trachea-esophageal fistula. Immediate attention has to be given for perforation since it may cause $50 \%$ mortality and emergency repair or flap coverage should be performed at the same setting.

\section{Preventing Vertebral and Carotid Artery Injuries}

- Injury to vertebral artery has an incidence of 0.3 to $0.5 \%$. Damage occurs most often during surgery anterior to the transverse foramen of $\mathrm{C} 7$ or during lateral compression maneuvers from C3 to C6. Careful identification of the longus colli and uncovertebral joints is crucial for midline dissections.

- As discussed above, the accurate dissection is warranted and retractors must be placed as mentioned and displacement anterolaterally can damage the carotid artery. This can be avoided by intermittent release of the retractors.

\section{Preventing Tracheal Injury}

- It is life-threatening as the defect may allow the esophagus to prolapse into the tracheal lumen, which cause acute asphyxia. It has to be repaired immediately with or without sternocleidomastoid muscle flap coverage.

- The injury can be prevented as placing the retractors carefully as discussed above and displacement anteromedially can compress the trachea medially.

\section{Preventing Thoracic Duct, Cervical Sympathetic Chain Injury}

- Thoracic duct injury can occur during left-sided neck dissection. The thoracic duct can be identified dorsal to the subclavian vein and should be protected. It may lead to chylorrhea and chylous fistula.

- The cervical sympathetic chain travels between the carotid sheath and longus colli. It should be preserved in mid-cervical approaches. Any retraction of dissection lateral to the longus colli can cause injury.

\section{Preventing Pharyngocutaneous Fistula}

This results from esophageal perforations during dissection, using a burr for decompression and during instrumentation of the anterior cervical spine.

\section{Preventing Spinal Cord and Nerve Roots Injuries}

- These injuries are caused during osteophyte removal with Kerrison rongeurs or by drilling. Meticulous hemostasis, adequate illumination and visualization, experience, and proper technique are important to avoid this complication. Spinal cord injury can also be caused by inserting the bone graft after discectomy. Proper appropriate sizing, shaping, and tapping the graft in place with proper depth and height can avoid bone graft extrusion.

- Electrophysiological monitoring using somatosensory and motor-evoked potentials of the spinal cord is useful for monitoring during surgery and is especially useful in highrisk patients who have preexisting cord contusion or severe stenosis. Preoperative baselines should be obtained in patients for comparison.

\section{Preventing Adjacent Segment Degeneration}

- This complication develops after $15 \%$ anterior cervical discectomy and fusion surgeries and $9 \%$ of all posterior surgeries. Fusion-less surgery is less likely to develop adjacent segment degeneration (ASD). Plating can hasten ASD if it is placed within $8 \mathrm{~mm}$ of the adjacent cranial disk segment and should be avoided.

\section{Preventing Pseudoarthrosis}

The incidence of pseudoarthrosis varies widely from 0 to $50 \%$. Surgeons should avoid prescription of drugs like non-steroidal antiinflammatory drugs, phenytoin, at least 10 weeks before surgery and avoid placing bone substitutes like bone morphogenic protein in fusion surgery in sensitive areas such as behind esophagus.

\section{CoRpectomy}

- The overall morbidity risk associated with corpectomy is 11 to $27 \%$. Unlike discectomy, corpectomies carry a greater risk of graft migration, strut graft dislodgement, infection, and pseudoarthrosis due to larger destabilization of the anterior column. Other complications include graft pistoning, mortise penetration, inadequate deformity correction, and failure of fixation and neurological compromise.

- Late complications include graft fracture, collapse or subsidence, and nonunion. Most graft dislodgement occurs soon after surgery (within 24 hours). Graft migration rates increase appreciably with each additional fusion level, with a reported odds ratio of 1.65. Supplemental external immobilization with a halo vest can theoretically increase the rigidity of the construct and decrease the chance of cage dislodgment, especially in long fusions.

- Graft displacement and subsidence are important problems with anterior surgery. Prevention requires good patient selection and surgical technique. Good preparation of the graft, obtaining parallel fusion beds, and sitting the graft under load from the cortical bone are important steps to ensure the maximum stability for fusion.

\section{Surgery for Ossified Posterior LONGITUDINAL LIGAMENT}

The reported complications after ossified posterior longitudinal ligament (OPLL) surgery are noteworthy: about 2 to $10 \%$ for quadriplegia and 5 to $17 \%$ for $C 5$ root injury. More lateral decompression is required as the OPLL expands laterally at the intervertebral disk level. This also carries the risk of injuring the vertebral artery if exploration is lateral to the uncinate process.

\section{Cervical Disk Arthroplasty/Replacement}

The general complications are similar between fusion and disk replacement. Patients with osteoporosis should avoid disk arthroplasties as good bone quality needed for tighter prosthesis fitting. Patients with facet degeneration and severe vertebral collapse should not have disk replacements performed. 


\section{Posterior Cervical Spine Surgery}

\section{Preventing Spinal Cord and Nerve Roots Injuries}

- The incidence is $0.18 \%$ and increases with severe cervical kyphosis correction (2.6\%). Late neurological complications can be avoided in posterior corrective surgery for cervical kyphosis by prophylactic foraminotomies in the presence of foraminal stenosis, kyphosis correction that does not exceed $9.7^{\circ} \mathrm{C}$ per spinal segment, and avoiding kyphosis correction at C4-C5, which is where the largest posterior shift of the spinal cord occurs leading to $C 5$ palsy. This is a common problem but most cases resolve spontaneously. Similar to anterior surgery, spinal cord monitoring is a good adjunct monitoring tool.

\section{Preventing C5 Palsy}

- It had been reported a $3.4 \%$ incidence of early postoperative C5 nerve root deterioration. These injuries are usually motordominant but may also have sensory and radicular pain. C5 dysfunction can occur immediately to 20 days postoperatively. Recovery usually occurs but take weeks, months, or as long as 6 years. Traction injuries to the nerve root are the likely cause due to the posterior shift of the decompressed cord. The $C 5$ root is at risk due to its direct and short course as it exits the spinal cord. It is also at the apex of lordosis. Hence, the distance of cord shift and root traction is greatest. With a posterior drift, C5 root tethering occurs and may be stretched beyond its tolerance limit. The deltoid has a single innervations by the $C 5$ nerve root, so any nerve dysfunction has a profound effect on patient function. Prophylactic foraminotomy should be considered in cases of preexisting deltoid weakness, intervertebral foraminal stenosis, OPLL, and laminectomy with instrumentation.

\section{Preventing Spring-back Closure}

- This complication has been reported at a rate of $40 \%$. This occurs only in suture fixation of laminoplasty.

\section{Preventing Postlaminectomy Kyphosis}

- The incidence of kyphosis deformity after multilevel laminectomy is $20 \%$. Older patients may have partially fused cervical spines and are more stable. Thus, postoperative kyphosis is more common in younger patients. Laminectomy should be avoided in young patients without cervical lordosis. Posterior facet joints should not be disrupted intraoperatively. Fusion should be considered for these patients at the same procedure.

\section{Screw Fixation in Cervical Spine}

- This technical expertise must be carefully done. Screw malposition varies from 0 to $4 \%$ in the atlas and 0 to $7 \%$ in the axis. Transarticular C1-C2 screws or Magerl screws pose an additional risk of vertebral artery injury, neurological deficit, or inadequate bony purchase. Vertebral artery injury is one of the most dangerous complications of screw fixation and is usually due to incorrect cervical pedicle screw entry with vertebral artery injury. The incidence of iatrogenic vertebral artery injury is 1.3 to $4 \%$ for Magerl fixation. Lateral deviation of screws can often lead to penetration of the foramen transversalis and subsequent vertebral artery injury. Current trends include the use of intraoperative CT and computer-assisted navigation systems to improve screw trajectory and reduce screw perforation.

- Subaxial lateral mass screws carry a risk of nerve root injury (1.3\%) and lateral mass fracture. Direct root injury during lateral mass screw insertion can be avoided with fluoroscopic control. Insertion under sagittal angulations of less than $15^{\circ}$ may lead to impingement of the exiting nerve root by the protruding screw threads. In axial trajectories greater than $30^{\circ}$ lateral to the midline, lateral mass fracture (1.6\%) or screw cut-out (1.3\%) may occur. However, neurological harm is not common. Screws placed too medially can cause vertebral artery injury.

\section{Posterior Occiptocervical InStRUMENTATION}

- In occipitocervical instrumentation, precise insertion of the occipital screws is crucial to prevent complications. Any screws inserted cephalad to the superior nuchal line can injure the transvers sinus. Loosening of screws is noted in $4.2-7 \%$ of cases and dural tears occur at a range from 0 to $4.2 \%$ during drilling of the occiput and screw placement. This area of the scalp is also quite then and skin erosions due to prominent implants can occur. Dural laceration can occur during burr hole drilling of the occiput or wire recoil with wire-based fixation (25-28\%). Dural laceration leads to CSF leak but screw insertions into the drill hole are usually sufficient to halt a CSF leak. Late complications of occipitocervical fusion include pseudoarthrosis (6\%) and adjacent level degeneration (7\%).

\section{Minimally Invasive Cervical Spine SURGERY}

- The limited visualization offered by minimally invasive spine surgery may lead to more severe complications. Manipulation in a tight neural foramen can cause root injury. Direct spinal cord injury may also occur during dilation or decompression. Using fluoroscopic image system, while inserting the blunt dilator, is advised to prevent it from entering the interlaminar space. This tactics prevents nerve injury. Dural tears have been reported in 1.6 to $6.6 \%$ of minimally invasive decompression procedures and conversion to open surgery is usually required to repair the dura.

\section{Vertebral Augmentation in Osteoporotic Fractures}

- In this unique procedure, patient selection and the radiographic evaluation are the most important criteria. The procedure to be done in the prone position, the vertebroplasty/kyphoplasty needle is placed in the vertebral body either through a transpedicular or parapedicular approach. Upmost care must be taken not to transverse the medial or inferior aspect of the pedicle, which may result in thecal sac, spinal cord damage, or nerve damage, respectively. ${ }^{5}$

\section{Pedicle Screw Fixation}

- This procedure is technically demanding and associated with a high complication rate. Screw misplacement (6.5\%) and screw 
breakage (12.4\%) inevitably lead to loss of correction. Inadequate fixation and subsequent motion also produce significant morbidity in patients. To avoid screw breakage and subsequent loss of correction, anterior support should be provided through either posterior or anterior lumbar interbody fusion (PLIF or ALIF) techniques in reduced spondylolisthesis L5-S1.

- The other important complication in pedicle screw fixation is nerve root irritation from medial angulation of the screw with resultant violation of the medial cortex of the pedicle.

- Optimal screw placement is typically along the medial aspect of the pedicle. The instrumentation gains purchase from its proximity to cortical bone but should not disrupt it; the tip of the pedicle screw should approach but not breach the anterior cortex of the vertebral body. Complications may arise from medial or lateral deviation of a screw or from its penetration of the anterior cortex of the vertebral body. The same complications arise from malpositioning of anterior cervical plates and screws, which may penetrate the adjacent disk space, foramen transversarium, spinal cord, or nerve roots. The graft material in either case may also be herniated anteriorly or posteriorly and cause neurologic compromise.

- The ideal pedicle screw should have a maximum diameter and length without breaching the pedicle's cortical layer or that of the vertebral body and it should converge.

\section{Preventing Deep Venous Thrombosis and Pulmonary Embolism}

Low-molecular-weight heparin regimes reduce the frequency of DVT and PE in spinal surgery. A mechanical prophylaxis also reduces the incidence of these dreadful complications.

\section{Preventing Surgical Site Infection in SPine Surgery}

- The incidence of surgical site infection (SSI) in spine surgery is 0.1 to $4.7 \%$. Staphylococcus aureus is the commonest organism involved. The risk may be due to patients factors like obesity, comorbid diseases like diabetes mellitus, immunosuppression, steroid usage, extremes of age or procedural factors like more operating time, more bleeding, more tissue trauma and more staff movements while operating.

- It is highly recommended to implement antibiotic stewardship program in to all the health establishments to prevent and manage the SSI. Tetanus prophylaxis to be given 48 hours before surgery. Proper education with regard to surgery and postoperative period. Antibiotic prophylaxis 15 to 30 minutes before surgery to be given. Either inj. cefuroxime $1.5 \mathrm{~g} \mathrm{IV} \mathrm{BD} \mathrm{for}$ 24 hours or inj. cefazolin $2 \mathrm{~g}$ IV BD for 24 hours and in implant surgery for 48 hours. Surgical site hair removal just before surgery (using clippers not razors) and chlorhexidine skin preparation. lodophor-impregnated drape, less tissue injury, and less diathermy usage.

- The anesthesiologist's role is also a key factor in preventing SSI and the following bundles with phase of care will help in producing the good results. Mitigating risk factors, glycemic control, maintaining normothermia, antibiotic prophylaxis, patient education..$^{6,18}$ The duration of surgery correlates with the bacterial load. More than $10^{5}$ organisms can be found in surgical wounds after 5.7 hours and operations longer than 3 hours increase the risk of infections. A good surgical technique is fundamental for reducing infection. This includes meticulous dissection within avascular planes and potential dead spaces.

\section{Discussion}

"To err is human," the report published by the Institute of Medicine (IOM) in the year 1999 drawn attention of the surgeons and public. In spite of the benefits of spine surgery for treating the appropriate pathologies, there are still many potential complications. It is calculated that the cumulative probability of having a wrong site surgery at least once in an orthopedic surgeon's life time carrier is $25 \%$. 2,6

The alertness of surgeons starts with the anesthesia and positioning to the surgical dissection, instrumentation techniques, and procedure. In anterior surgery, avoiding prolonged and forceful retractions can prevent injury to the esophagus, recurrent laryngeal nerve, and carotid arteries. Additional protection of corpectomies with plating combined with posterior spinal fusion and instrumentation can help reduce rates of graft dislodgements. Careful selection of patients for corpectomy is also important as graft collapse and subsidence commonly occurs with osteopenic bone. In posterior surgery, prophylactic foraminotomy may help reduce the risk postoperative C5 nerve root palsy. Spinal cord monitoring is advocated in all cases of cervical spine surgery. Careful analysis of the bony and vascular anatomy should be done preoperatively, especially when internal fixation is contemplated. Preservation of posterior muscle and their attachments are important for prevention of postoperative neck pain and delayed kyphosis. Most complications are manageable with adequate preparation. Applying principles of antisepsis will prevent surgical site infection. When carefully and properly executed, cervical spine surgery can be effective with an acceptable rate of complications. Complications of spine surgery may be difficult to diagnose and it is frequently difficult to identify the causes of recurrent symptoms on clinical grounds alone. Radiography is the standard follow-up imaging method..$^{2,6}$

Complications are the main concern of patients and surgeons at the time of spine surgery indication and when they occur, it will lead to personal and economic consequences affecting the quality of life and future independence of patients. Studies have shown that surgeons and gynecologists have a lower life expectancy than clinicians and emotional stress may be a cause of this difference. Surgical complications are a relevant cause of stress in surgeons. We need to take upmost care in preventing these technical hitches considering patients and our lives involved. The North American Spine Society (NASS) had developed a sign, mark, and X-ray (SMax) program for identification of exact patient and operation level. This program has three key factors for patient safety in spine surgery:

- Process of patient identification and confirmation of medical records including imaging studies and informed consents.

- Surgical site markings.

- Verification of the spine pathology level during operation using radiography.

In 2008, the World Health Organization (WHO) had introduced guidelines for the safety of surgical patients. Since the use of WHO guideline checklists in the hospitals around the world, the mortality rate has declined to $0.8 \%$ from $1.5 \%$.The patient-related complications have also reduced to $7.0 \%$ from $11 \% .{ }^{6,10}$ The key 
points in reducing complications are the surgeon's familiarity with operative imperatives and the appropriate surgical approach. ${ }^{2,6,10}$

\section{Conclusion}

Even with the fact that the surgical treatment for all spine diseases and fractures is acceptable at the moment, the orthopedic surgeon must ensure the upmost safety of the patient well during surgery.

Following WHO guidelines of safe surgery in patient safety is essential in all health establishments. To evade the serious complications, the applied anatomical knowledge with expert skills is essential for the surgeon while carrying out spine surgery.

\section{Ethical Approval}

This article does not contain any studies with human participants or animals performed by any of the authors.

\section{References}

1. Jutte PC, Castelein RM. Complications of pedicle screws in lumbar and lumbosacral fusions in 105 consecutive primary operations. Eur Spine J 2002;11(6):594-598. DOI: 10.1007/s00586-002-0469-8.

2. Young PM, Berquist TH, Bancroft LW, et al. Complications of spinal instrumentation. Radiographics 2007;27(3):775-789. DOI: 10.1148/ rg. 273065055 .

3. Proietti L, Scaramuzzo L, Schiro G, et al. Complications in lumbar spine surgery; a retrospective analysis. Indian J Orthop 2013;47(4):340-345. DOI: 10.4103/0019-5413.114909.

4. Chahoud J, Kanafani Z, Kanj SS. Surgical site infections following spine surgery: eliminationg the controversies in the diagnosis. Front Med 2014;1:7. DOI: 10.3389/fmed.2014.00007.

5. Barrocas AM, Eskey CJ, Hirsch JA. Vertebral augmentation in osteoporotic fractures, injury. Int J Care Injured 2007;3853:588-596.

6. Lee $\mathrm{S}-\mathrm{H}$, Kim J-S, Jeong $\mathrm{Y}-\mathrm{C}$, et al. Patient safety in spine surgery; regarding the wrong-site surgery. Asian Spine J 2012;7(1):62-71.
7. Gautschi OP, Schatlo B, Schaller K, etal.Clinically relevant complications related to pedicle screw placement in thoracolumbar surgery and their management; a literature review of 35630 pedicle screws. Neurosurg Focus 2011;31(4):E8. DOI: 10.3171/2011.7.FOCUS11168.

8. Licina P, Nowitzke AM. Approach and considerations regarding the patient with spinal injury, injury. Int J Care Injured 2005;36(Suppl. 2):B2-B12. DOI: 10.1016/j.injury.2005.06.010.

9. Fehlings MG, Perrin RG. The role and time of early decompression for cervical spinal cord injury; update with a review of recent clinical evidence. Int J Care Injured 2005;36(Suppl. 2):B13-S26. DOI: 10.1016/j. injury.2005.06.011.

10. WHO guidelines for safe surgery: safe surgery saves lives, 2009 .

11. Nasser R, Yadla S, Maltenfort MG, et al. Complications in spine surgery. J Neurosurg: Spine 2010;13(2):p1-p5. DOI: 10.3171/2010.3.SPINE09369.

12. He B, Yan $L, X u Z$, et al. Treatment strategies for the surgical complications of thoracic spinal stenosis: a retrospective analysis of two hundred and eighty three cases. Int Orthop 2014;38(1):117-122. DOI: $10.1007 / \mathrm{s} 00264-013-2103-2$.

13. Antimicrobial stewardship: systems and processes for effective antimicrobial medicine use (2015), NICE Guidelines, UK.

14. Reis RC, Oliveira MF, Rotta JM, et al. Risk of complications in spine surgery: a prospective study. Open Orthop J 2015;9(1):20-25. DOI: 10.2174/1874325001509010020.

15. Cheung JPY, Dip-Kei Luk K. Complications of anterior and posterior cervical spine surgery. Asian Spine J 2016;10(2):385-400. DOI: 10.4184/ asj.2016.10.2.385.

16. National Treatment Guidelines for antimicrobial use in infectious diseases, National Centre for Disease control, Directorate General of Health Services, Ministry of Health \& Family welfare, Government of India, 2016.

17. Treatment Guidelines for Antimicrobial use in common syndromes, Indian Council of Medical Research, Department of Health Research, New Delhi, India 2017.

18. Seligman KM, Kotz D, Farber MK. Preventing Surgical Site Infection after Cesarean Delivery-The Anaesthesia Professional's role, APSF News Letter, June 2018, pp. 25-27. 